\title{
Some Questions of the Mixed Anxiety-Depressive Disorders in Patients with Cerebellar Stroke in the Early Period of Convalescence
}

\author{
V. A. Kutashov, PhD, ScD; O. V. Ulyanova, PhD; I. S. Protasov, PhD; \\ A. P. Skorokhodov, PhD, ScD; O. V. Zolotaryov, PhD; L. S. Nemykh, PhD; \\ E. S. Ananyeva, PhD; A. A. Dudina, PhD; M. V. Uvarova \\ Voronezh State Medical University named after N.N. Burdenko \\ Voronezh, the Russian Federation
}

\begin{abstract}
Background: The objective of this study was to investigate the medico-social and mixed anxiety-depressive disorders (MADD) in patients with cerebellar stroke (CS) in the early recovery period.

Methods and Results: The study included 140 patients (87/62\% men and 53/38\% women) with average age of $57.2 \pm 2.2$ years. Patients were examined in the early recovery period ( 6 months from the onset of the disease). The level of anxiety and depression was assessed using the Hamilton Rating Scale for Depression. In the early recovery period from CS, the difeferent types of MADD were diagnosed. A high level of depression was found in $3.57 \%$ of patients, the average level - in $27.86 \%$, mild depression - in $33.57 \%$ of patients, and the absence of depressive symptoms in $35 \%$ of patients. A high level of anxiety in $17.50 \%$ of CS patients, a moderate level of anxiety in $47.86 \%$, a low anxiety level in $45.0 \%$, and no anxiety in $2(1.43 \%)$ patients.

Conclusion: Different levels of MADD in CS patients in the early period of convalescence need individual specific treatment. (International Journal of Biomedicine. 2020;10(2):108-111.)
\end{abstract}

Key Words: acute cerebrovascular accident, cerebellar stroke, mixed anxiety-depressive disorders

\section{Abbreviations}

BP, blood pressure CS, cerebellar stroke; HRDC, the Hamilton Rating Scale for Depression; HT, hypertension; MADD, mixed anxiety-depressive disorders.

\section{Introduction}

More than 450,000 cases of stroke are registered in Russia annually. The incidence of acute cerebrovascular accident in Russia is $2.5-3.52$ cases per 1000 population per year, and mortality in the acute period of stroke reaches $35 \%$, increasing by $12 \%-15 \%$ by the end of the first year; within 5 years $44 \%$ of stroke patients die. The highest mortality rate is recorded for acute strokes in the carotid artery supply $(60 \%$ during the first year). ${ }^{(1-4)}$

*Corresponding author: Igor S. Protasov, PhD. Voronezh State Medical University named after N.N. Burdenko. Voronezh, Russia.E-mail:protasovis@mail.ru
Cerebellar stroke (CS) is a relatively rare cerebrovascular pathology. It accounts for $1.5 \%$ to $2.3 \%$ of all acute cerebrovascular accidents. The signs and symptoms of different CSs are quite familiar to neurologists. Rehabilitation prognosis is also better than in patients who have strokes in the carotid supply or in the brain stem. ${ }^{(1-10)}$ However, there is insufficient data about the recovery of patients with CS, taking into account the peculiarities of medical and social characteristics and the presence of affective and anxiety disorders. CS is a sudden and serious illness, which can cause psychological trauma and is accompanied by a neurotic, hypochondriac and depressive reaction. In this context, great importance is given to the study of personal characteristics of patients who have undergone $\mathrm{CS}$, in order to determine 
personality traits that inhibit the formation of a positive attitude to treatment. ${ }^{(1,3,4,5,7,9,10)}$

According to several studies, among patients with CS, 25\% have serious limitations that affect their everyday life. First of all, they need rehabilitation. A major factor affecting the result of the treatment and rehabilitation is the formation of complex psychopathological conditions. ${ }^{(7-10)}$ Often, psychiatric pathology advocates for a significant factor in the restoration of destroyed social functions-household adaptation. Due to the development of stroke, the most common psychiatric disorder is mood changes, the prevalence of which varies from $30 \%-60 \%$. Recently, MADD as a form of affective disorders is attracting more and more attention, not only from psychiatrists, but also from neurologists since the combination of MADD and somatic pathology is included among the factors that adversely affect the treatment process and rehabilitation. ${ }^{(3,5,7,9)}$

The objective of this study was to investigate the medicosocial and mixed anxiety-depressive disorders in patients with cerebellar stroke in the early recovery period.

\section{Materials and Methods}

The study was conducted in accordance with ethical principles of the WMA Declaration of Helsinki (1964, ed. 2013) and approved by the Ethics Committee of Voronezh State Medical University. Written informed consent was obtained from all participants.

Our study included patients with CS verified by modern diagnostic methods and undergoing treatment from 2013 to 2019 in the Voronezh Regional Clinical Hospital \#1, and City Emergency Medical Hospitals \#1 and \#10. The study was prospective in character; it included patients with a favorable course of the disease. The study included 140 patients $(87 / 62 \%$ men and 53/38\% women) with average age of $57.2 \pm 2.2$ years. Patients were examined in the early recovery period ( 6 months from the onset of the disease). In the earliest period of convalescence, all CS patients were assessed for a stroke scale, and given a neurological examination and psychometric testing. The level of anxiety and depression was assessed using HRDS.

The causes of CS were often several factors: HT, atrial fibrillation, atherosclerosis of cerebral arteries, degenerativedystrophic changes in the cervical spine, and compression of the vertebral arteries.

Statistical analysis was performed using the Statistica 6.1 software package (Stat-Soft Inc., USA).

\section{Results}

Duration of illness when the study started was on average $39.0 \pm 8.0$ days. Nine $(6.4 \%)$ patients had relatives who have undergone a cerebrovascular accident. We analyzed the features of patients after CS: $50 \%$ of all patients live with spouse and children, $27.2 \%$ with relatives, and $22.8 \%$ live alone. Only $20 \%$ of patients were not satisfied with their housing conditions, such as the small size of their housing, lack of water supply, crowded housing, and poor heating system.
Most of the patients suffered from HT. However, only $25.5 \%$ of patients were monitored daily for BP; $17.8 \%$ were monitored several times in a week, and $17.8 \%$ - several times in a month. Moreover, $16.4 \%$ of patients were forced to measure BP in medical institutions, since they did not have a personal BP monitor; $26.4 \%$ of patients did not measure BP at all, and $16.4 \%$ measured BP 1 time per year. An analysis of indicators characterizing compliance to HT therapy showed that $59(58.4 \%)$ patients out of 101 who were prescribed antihypertensive therapy, for various reasons, would skip prescribed medicine. It was found that $65.7 \%$ of patients have never obtained special training for patients with HT and other vascular problems; at the same time $47.8 \%$ of people admitted that they lacked information about their previous disease conditions. Despite ongoing measures to provide information for the population to prevent the development of stroke, some patients did not receive the necessary information or did not receive it in full format.

Before CS development, $92.9 \%$ of patients had complaints, yet $26.4 \%$ of them did not see a doctor for help, and did not control the BP level, even with the following symptoms: high BP, headache, chest pain, dizziness, tinnitus, hearing loss, and slight numbness or weakness in the limbs. Thus, it can be concluded that the therapeutic compliance prior to the development of CS was very low: patients took drugs irregularly and did not follow any diagnostic or preventive regimen. Moreover, most patients had one or more complaints preceding the development of CS.

In the early recovery period from $\mathrm{CS}$, the following types of MADD were diagnosed: $20 \%$ of patients with an ergopathic and $15.7 \%$ of patients with a hypochondriac type of relationship to the disease. Less often were neurasthenic $(8.5 \%)$ and sensitive (10\%) types. A small percentage of patients, $7.1 \%$, were identified with harmonious, anosognostic, and anxious types of attitudes towards the illnesses. We further identified $5.7 \%$ of the egocentric type, $6.4 \%$ each of the paranoid and depressive types, and $2.8 \%$ of the melancholic and apathetic types of relationship to the disease.

Based on the analysis, we can conclude that the observed patients with CS were predisposed to such personality traits as neurotic response, low self-esteem, anhedonia, and depression, correlated with personal characteristics, stress response to ordinary life situations that proceeds according to a passivedefensive type. Identified: anxiety, abstinence, and lack of confidence. As a result, difficulties arise in forming social contacts, as well as a decrease in the degree of spontaneous aggression, which most often indicates difficulties in expressing negative emotions. Low grades on the scale of "sociability" and "extraversion-introversion" indicate that this group of patients had low social activity. Based on the received results it can be concluded that the observed CS patients revealed a low level of quality of life, as well as a non-adaptive type of relation to the disease, in view of the presence of personality traits predisposing them to neurosis and stress response.

The most common complaints in the psycho-emotional sphere that were presented by CS patients in the early recovery period were decreased mood, tearfulness, and increased 
fatigue. Often, patients complained of sudden mood swings, excitement, anxiety at the slightest occasion and without reason, difficulty or impossibility of performing daily activities, decreased concentration, obsessive self-derogatory thoughts, disturbed sleep, anxiety about the future, thoughts of suicide, and thoughts of impending death. All patients experienced manifestations of general physical and mental weakness (asthenia). These complaints prevailed in the clinical picture. Patients complained of fatigue that did not go away after rest, for both long and short periods of time, general weakness, lack of desire to deal with any everyday issues, and incredibly rapid exhaustion. Most patients refused to perform any kind of hard and complex work. Only 17(12.4\%) patients decided to continue work but transferred to another position, in connection with expressed asthenic phenomena to a great extent. Patients who potentially preserved working capacity really wanted to work. They wanted to be helpful by applying extraordinary efforts to do any kind of work. A number of patients felt guilty for forced idleness and considered themselves an excessive burden on the family, which undoubtedly worsened their psycho-emotional status. Very often, patients complained of a reduced need for communication, a weak desire to maintain mutual relations with friends, difficulties in communicating with loved ones and colleagues. Many patients wanted to stay alone longer, explaining that they lacked the strength to see anyone. Many patients had emotional lability in the form of "inability to control emotions."

When conducting a special study, we encountered resistance to our efforts. Patients reluctantly agreed to psychological testing. Many patients tried to embellish their subjective state. The majority spoke negatively of psychiatry and psychological help.

Patients maximally fixed their attention on the smallest manifestations of CS. Many of the patients significantly exaggerated the significance and severity of neurological and other somatic disorders. A number of patients insisted to doctors that they "did not correctly establish the diagnosis." Many spoke out that the disease is very severe, and the treatment "cannot change anything, the outcome is clear." In the hospital, patients believed that the doctors did not pay enough attention to their "incurable disease," and that doctors prescribe drugs that are too "simple, cheap" or the completely wrong treatment. Many patients and their relatives in the early recovery phase of CS turned to alternative medicine. Patients expressed that they do not rely on the effectiveness of academic treatment.

Based on the results obtained, the presence of MADD in patients with CS was revealed in the early days of the recovery period. According to the results obtained during the initial psycho-diagnosis, according to the HRSD, a high level of depression was found in $3.57 \%$ of patients, the average level - in $27.86 \%$, mild depression - in $33.57 \%$ of patients, and the absence of depressive symptoms in $35 \%$ of patients.

Thus, it can be noted that with minimal and moderate depression, patients experience a dreary mood, a feeling appears that their life is at an impasse, and they experience bad feelings in the soul. They see the bad slightly more than usual in their past, thus hope for the best decreases, there appears the fear that there are no reasons for joy, they do not enjoy life as before, and they feel that life is meaningless. However, with mild to moderate depression, these conditions pass more quickly; patients can independently cope with them, find positive moments in life, and return to the normal course of their lives. The situation is different for people with severe depression. Such patients note that almost all the time they have bad feelings in the soul, they worry about their future, and they lose hope for the best. They constantly feel like their life is meaningless, noting that this condition never goes away. They see in themselves and others only the bad; they are constantly irritable, have no strength to do anything, no appetite, and a constant desire to cry. A depressed mood is regular for them, and in the morning, it can be worse than in the evening. It is very difficult to cope with a depressed status; it can last a long time and can develop into a normal state of constant depression.

So, the study showed that deep depressive states were not inherent in a significant number of studied patients.

A study of anxiety levels showed a high level of anxiety in $17.50 \%$ of CS patients, a moderate level of anxiety in $47.86 \%$, a low anxiety level in $45.0 \%$, and no anxiety in $2(1.43 \%)$ patients.

As we can see, patients who have undergone CS are characterized by a moderate level of anxiety. This level of anxiety indicates that different life situations tend to be perceived adequately, with the subject objectively assessing the current situation. Such subjects are confident enough in most life situations; they feel calm, collected. They are not inclined to avoid critical situations and difficulties. However, they may have periods when they greatly experience their frustrations and cannot forget about them for a long time. In such moments the subjects lack self-confidence and the level of personal anxiety may rise. In general, these subjects can be described as calm, balanced, not prone to excessive irritability and anxiety.

For subjects with a high level of anxiety, it is characteristic to have excessive anxiety in a wide range of life situations. They are afraid of all sorts of trifles and minor troubles. In situations that are not even significant, they do not feel confident and are not able to maintain balance. The occurrence of anxiety is possible even in minor situations; they rarely feel calm, collected and confident. They tend to avoid critical situations and difficulties, believing that they do not have enough strength to deal with them. Generally such patients can be described as disturbed, irritable, rarely calm and balanced, and prone to excessive anxiety. Thus, the obtained indicators characterize them as prone to exhibit excessive anxiety, worried and distracted by trifles and minor troubles. They rarely feel calm, collected and confident, and often try to avoid critical situations and difficulties, believing that they do not have enough strength to deal with them.

\section{Discussion}

Our study found that a high level of depression was inherent in only a small number of subjects (3.57\%). Mild or moderate depression was more characteristic of the patients 
( $33.57 \%$ and $27.86 \%$, respectively). With mild to moderate depression, these conditions pass quickly; subjects can independently cope with them, find positive moments in life, and return to the normal course of their lives.

Patients with severe depression note that they have a bad feeling in the soul almost all the time, they worry about their future, and do not have hope for the best things. They constantly feel the meaninglessness of their life and see only the bad things for themselves and others. They are constantly irritable and have no strength to do anything, no appetite, and a constant desire to cry.

Most patients (47.86\%) with CS have a moderate level of anxiety. Such subjects are quite confident in themselves; in most of life situations they feel calm, collected. But they can have periods when they greatly experience frustrations and cannot forget about those feelings for a long time. In such moments, these patients lack self-confidence and the level of personal anxiety may rise.

A high level of anxiety occurred in $17.50 \%$ of patients with CS; the results characterize them as prone to be overly anxious, worried and distracted by trifles and minor troubles. They rarely feel calm, collected and confident, and are often inclined to avoid critical situations and difficulties, believing that they do not have enough strength to deal with them. Thus, the results obtained indicate that patients with CS, at the stage of recovery, have a high level of anxiety and depressive manifestations. That finding determines the need for early detection and prescription of specific therapy for anxiousaffective pathology in CS. Different levels of MADD in CS patients in the early period of convalescence need individual specific treatment.

\section{Competing Interests}

The authors declare that they have no competing interests.

\section{References}

1. Stroke: A guide for doctors. Ed. Stakhovskaya LV, Kotov SV. M.:LLC Medical News Agency;2013:400 pp. [In Russian] 2. Stroke: diagnosis, treatment, prevention. Ed. Suslina ZA, Piradov MA. M.: MED Press-Inform; 2008:288 pp. [In Russian].

3. Kutashov VA, Ulyanova OV. [The most significant predictors of cardio-embolic stroke, which determine the clinical picture and the outcome of the disease]. Bulletin of Neurology, Psychiatry and Neurosurgery. 2016;(6):51-55. [Article in Russian].

4. Kutashov VA, Budnevsky AV, Ulyanova OV, Sazonov IE, Khabarova TY. [Modern therapy of cerebrovascular diseases in the Voronezh region]. Bulletin of Medical Internet conferences. 2016;6(10):1543. [Article in Russian].

5. Skvortsova VI. Fundamentals of early rehabilitation of patients with acute impairment of cerebral circulation. M.:MODEK; 2006:336 pp. [In Russian].

6. Ulyanova OV, Kutashov VA. [On the issue of cardiogenic risk factors for the onset of ischemic stroke in young adults]. Cardiovascular Therapy and Prevention. 2015;1(15):62-63. [Article in Russian].

7. Vein AM, Voznesenskaya TG, Golubev VL, et al. Depression in neurological practice (clinic, diagnosis, treatment). 3rd ed. M.:MIA;2007:208 pp. [In Russian].

8. Depression and comorbid disorders. Collective monograph edited by A. Smulevich. M.: Enlightenment; 1997:426 pp. [In Russian].

9. Surzhko GV, Kutashov VA, Khabarova TB, Ulyanova OV. Psycho-correction of anxiety - depressive disorders in patients with stroke in the early recovery period. Voronezh; 2017:170 pp. [In Russian].

10. Shklovskiy VM. The concept of neurorehabilitation of patients after a stroke. Journal of Neuropathology and Psychiatry named after S.S. Korsakov: Appendix. Stroke. 2003;(8):77-81. [Article in Russian]. 